\title{
Seasonal Onychomadesis of the Great Toes
}

\author{
Hadley J. Pearson ${ }^{a}$ Robert T. Brodell ${ }^{b, c}$ C. Ralph Daniel III ${ }^{b, d}$ \\ a University of Mississippi Medical School, and Departments of ${ }^{b}$ Dermatology and ${ }^{\mathrm{c} P a t h o l o g y}$, University of Mississippi \\ Medical Center, Jackson, MS, and d Department of Dermatology, University of Alabama, Birmingham, AL, USA
}

\section{Established Facts}

- Nail trauma is a common cause of onychomadesis.

\section{Novel Insights}

- Because of the slow growth of toenails there is a delay between insult and identifiable nail changes. Recalling the causative insult in onychomadesis can be challenging for patients.

- The pathogenesis of onychomadesis should be reviewed with the patient to enable them to help identify subtle behavioral factors that may contribute to their disease.

\section{Keywords}

Diagnosis · Nail · Nail disorder · Nail pathology

Onychomadesis

\begin{abstract}
Onychomadesis occurs when the proximal nail plate becomes unattached to the underlying structures. When only one or a few nails are involved, the cause is usually physical trauma. A 23-year-old female presented with a 4-year history of recurrent onychomadesis involving her bilateral great toes that occurred during the springtime. While recurrent trauma from winter shoes or athletic activities may be a contributing factor, our patient was convinced that wearing tight-fitting socks during winter nights was the primary pathophysiologic process. By wearing socks only during the days and decreasing the amount of time the nails spent traumatized due to pressure, the patient's recurrent condition was eliminated.

(c) 2017 S. Karger AG, Basel
\end{abstract}

\section{KARGER}

(c) 2017 S. Karger AG, Basel

E-Mail karger@karger.com

www.karger.com/sad

\section{Introduction}

Onychomadesis occurs when the proximal nail plate is separated from the underling matrix with persistent attachment to the distal nail bed. As the distal portion of the nail grows out, the separated proximal portion is painlessly shed [1]. The pathogenesis of onychomadesis is similar to that of Beau's lines where transverse depressions result from a thinned nail plate due to slowing or disruption of cell growth within the matrix [2].

A variety of causes of Beau's lines and onychomadesis have been identified: trauma, infectious disease, autoimmune, neonatal, systemic disease, hereditary, medication induced, and idiopathic [2]. In all of these conditions, an insult to the nail matrix results in an alteration of nail growth. Since many factors can disrupt the matrix, proper diagnosis depends on a careful history and physical examination. 


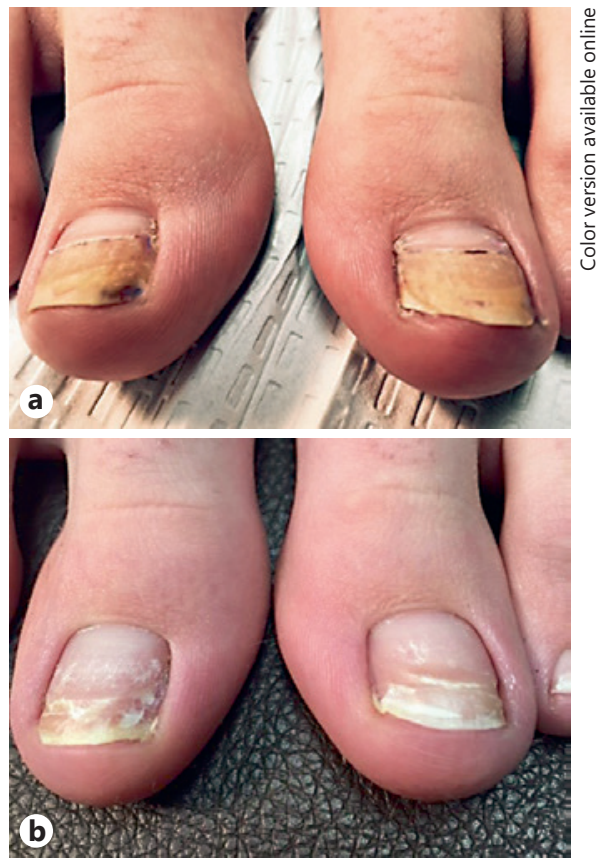

Fig. 1. Progression of nail growth over a 6-month time period. a Onychomadesis 2 months after onset of pain and erythema at the proximal nail fold. $\mathbf{b}$ Six months later, the distal nail has been shed revealing the underlying depressed nail plate.

The distribution of the onychomadesis can provide clues as to the cause, with disease affecting multiple digits concurrently more likely attributable to a systemic process such as hand-foot-mouth disease, Stevens-Johnson syndrome, lichen planus, a drug side effect, or several other diseases [3]. When the process is isolated to a single digit, local trauma is the most likely etiology [4]. The occurrence of pain preceding onychomadesis should prompt consideration of either an inflammatory or traumatic cause $[3,5]$. The timing of the first instance of nail dystrophy is also significant: persistent dystrophy of the bilateral great toenails, especially if present since childhood, may be due to congenital malalignment of the great toenails. In this condition, the longitudinal axis of the nail matrix is laterally deviated and continuous microtrauma causes the nail to grow out in thickened ridges [6]. Recurrent onychomadesis is uncommon and suggests temporally separated, repetitive matrix insults. Some recurrent cases have been attributed to autoimmune disease relapses or reported as familial or idiopathic [7]. A patient with recurrent seasonal onychomadesis of the great toenails is reported.

\section{Case Report}

A 23-year-old white female college student presented with a 4 -year history of recurrent yellowing and detachment of the great toenails bilaterally. No other nails had been affected. Each episode occurred in the springtime and was associated with pain and redness at the base of the toenails, followed several weeks later by nail changes. Normal nail growth occurred during the rest of the year. A 12-week course of oral terbinafine had no effect on these cyclic nail changes. She denied any recent illnesses or medication use. No family members reported similar symptoms.

On physical examination, the distal nails of the bilateral great toes were dull, yellow, and separated from the healthy proximal nail (Fig. 1a). All other toenails and fingernails were normal with no associated skin changes. $\mathrm{KOH}$ and fungal culture were both negative. Given the bilateral distribution and seasonal recurrence, we suspected recurrent nail trauma due to winter footwear. Six months later, the patient was seen again. The dystrophic nail had been shed, leaving behind a thinned nail plate with normal proximal nail growth extending halfway along the nailbed (Fig. 1b).

Examination of the patient's shoes revealed wide toe boxes with no temporal linkages between purchase and onset of symptoms. She was advised to avoid tight fitting shoes and nail trauma. In the summer of the following year, the toenails had returned to normal without any new onychomadesis. The only behavioral change that correlated with the resolution of onychomadesis was the discontinuation of nighttime sock wearing during the winter months.

\section{Discussion}

This case is remarkable because of its seasonal recurrence and localization to the great toes. Only one previous report of seasonal onychomadesis was identified: a 79-year-old man with a 10-year history of idiopathic recurrent Beau's lines and onychomadesis of all fingernails, occurring in the winter [8]. Additionally, a single case of idiopathic recurrent onychomadesis of the toenails, not attributable to systemic disease or familial inheritance, has been reported: a 38 -year-old Thai female with a 10 -year history of sporadic recurrence involving all toenails [9].

Traumatic onychomadesis is generally associated with obvious, sometimes dramatic trauma, such as repetitive friction from long-distance running, wrist and arm fracture, or fingertip crush injury $[2,10]$. The use of ill-fitting, closed-toe shoes with a narrow toe box in the winter is a logical source of recurrent, seasonal toenail trauma. Our patient, however, denied wearing any uncomfortable shoes correlating with the onset or resolution of the onychomadesis. The patient believed that wearing cotton socks overnight caused transferred pressure to the proximal nail fold, with the following factors contributing: the patient has an "Egyptian foot," i.e. the great toe is the longest digit with the second through fifth digits decreasing 
in length. A foot with Morton's toe (a long second toe) is known as a "Greek foot." These foot shapes refer to regional differences in the artistic depiction of feet found on ancient statuary. In the Egyptian foot, the long first toe is subjected to the greatest amount of mechanical pressure from tight socks. The sock exerts a downward force on the distal nail plate. With the nail bed as the fulcrum, a lever effect lifts the nail over the nail matrix.

The patient's large feet (size 10.5 in shoes) were compressed by standard women's socks made to fit sizes 5-9.

Most importantly, the patient started wearing socks during winter nights 4 years previously when she moved into a cold college dormitory. She suspected the socks as a causative factor in early winter soon after starting to wear socks at night and noticing pain at the proximal nail fold in the morning. She recalled a similar pain in previous years when wearing socks to bed. When she stopped wearing socks overnight, the pain resolved and the onychomadesis failed to recur.

If educated on the etiology of their condition, it is possible that other patients' idiopathic onychomadesis could benefit from similar, self-identified behavioral changes.

\section{Statement of Ethics}

The patient was seen as part of routine care and she gave informed consent for use of the photographs

\section{Disclosure Statement}

Robert T. Brodell, MD, discloses the following potential conflicts of interest: clinical trials have been performed for Genentech and Janssen Biotech, Inc. C. Ralph Daniel III, MD and Hadley J. Pearson have no conflicts of interest.

\section{Author Contributions}

Hadley J. Pearson performed initial literature search and wrote the first draft of the manuscript. C. Ralph Daniel III and Robert T. Brodell reviewed and approved the content of the final draft. This article has not been previously published in the literature.

\section{References}

1 Hardin J, Haber RM: Onychomadesis: literature review. Br J Dermatol 2015;172:592-596.

2 Braswell MA, Daniel CR, Brodell RT: Beau lines, onychomadesis, and retronychia: a unifying hypothesis. J Am Acad Dermatol 2015; 73:849-855.

3 Salgado F, Handler MZ, Schwartz RA: Shedding light on onychomadesis. Cutis 2017;99: 33-36.

4 Clark CM, Silverberg NB, Weinberg JM: What is your diagnosis? Onychomadesis following hand-foot-and-mouth disease. Cutis 2015;95:312, 319-320.
5 Kim HS, Lee SH, Yoon TJ, Oh CW, Kim TH: Early stage onychomadesis presenting as painful swellings of proximal nail folds. Cutis 2001;67:317-318.

6 Decker A, Scher RK, Avarbock A: Acquired congenital malalignment of the great toenails. Skin Appendage Disord 2016;1:147-149.

7 Hardin J, Haber RM: Idiopathic sporadic onychomadesis: case report and literature review. Arch Dermatol 2012;148:10-11.
8 Venugopal SS, Murrell DF: Seasonal onychomadesis in an elderly gentleman. Dermatol Reports 2010;1:e3.

9 Suchonwanit P, Nitayavardhana S: Idiopathic sporadic onychomadesis of toenails. Case Rep Dermatol Med 2016;2016:6451327.

10 Purim KSM, Leite N: Sports-related dermatoses among road runners in Southern Brazil. An Bras Dermatol 2014;89:587592. 